\title{
THE CONVEXITY AND THE CONCAVITY DERIVED FROM NEWTON'S INEQUALITY
}

\author{
Xun-Tuan Su AND WEI-Wei ZHANG
}

Abstract. By Newton's inequality, a sequence $\left\{a_{i}\right\}_{i=0}^{n}$ of nonnegative real numbers is unimodal if its generating function $\sum_{i=0}^{n} a_{i} x^{i}$ has only real zeros. This paper is devoted to show that there exist two indices $s$ and $t$ with $s \leqslant t$, such that $a_{0}, a_{1}, \ldots, a_{s-1}, a_{s}$ and $a_{t}, a_{t+1}, \ldots, a_{n}$ are convex, while $a_{s-1}, a_{s}, \ldots, a_{t}, a_{t+1}$ is concave.

Mathematics subject classification (2010): 05A10, 05A20.

Keywords and phrases: convexity, concavity, strict log-concavity, Newton's inequality.

\section{REFERENCES}

[1] E. A. Bender, Central and local limit theorems applied to asymptotic enumeration, J. Combin. Theory Ser. A 15 (1973), 91-111.

[2] F. Brenti, Unimodal, log-concave, and Pólya frequency sequences in combinatorics, Mem. Amer. Math. Soc. 413 (1989).

[3] F. BRENTI, Log-concave and unimodal sequences in algebra, combinatorics, and geometry: An update, Contemp. Math. 178 (1994), 71-89.

[4] G. H. Hardy, J. E. Littlewood, G. Pólya, Inequalities, Cambridge Univ. Press, Cambridge, 1952.

[5] L. H. HARPER, Stirling behavior is asymptotically normal, Ann. Math. Statist. 38 (1967), 401-414.

[6] P. H. Lundow, K. MARKSTRÖM, Broken-cycle-free subgraphs and the log-concavity conjecture for chromatic polynomials, Experiment. Math. 15 (2006), 343-353.

[7] R. P. STANLEY, Log-concave and unimodal sequences in algebra, combinatorics and geometry, Ann. New York Acad. Sci. 576 (1989), 500-534. 